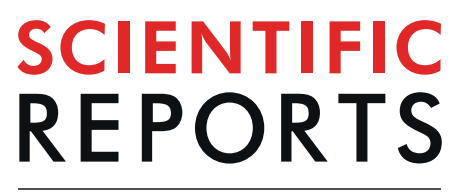

natureresearch

\title{
OPEN Prehospital lactate improves prediction of the need for immediate interventions for hemorrhage after trauma
}

Received: 13 February 2019

Accepted: 16 August 2019

Published online: 24 September 2019

\author{
Hiroshi Fukuma ${ }^{1}$, Taka-aki Nakada $\mathbb{1}^{1,2}$, Tadanaga Shimada ${ }^{2}$, Takashi Shimazui ${ }^{2}$, \\ Tuerxun Aizimu ${ }^{3}$, Shota Nakao ${ }^{1}$, Hiroaki Watanabe ${ }^{1,4}$, Yasuaki Mizushima ${ }^{1}$ \& \\ Tetsuya Matsuoka ${ }^{1}$
}

The blood lactate level is used to guide the management of trauma patients with circulatory disturbance. We hypothesized that blood lactate levels at the scene (Lac scene) could improve the prediction for immediate interventions for hemorrhage. We prospectively measured blood lactate levels and assessed retrospectively in 435 trauma patients both at the scene and on arrival at the emergency room (ER) of a level I trauma center. Primary outcome was immediate intervention for hemorrhage defined as surgical/radiological intervention and/or blood transfusion within $24 \mathrm{~h}$. Physiological variables plus Lac scene significantly increased the predictive value for immediate intervention (area under the curve $[A \cup C] 0.882,95 \%$ confidence interval $[\mathrm{Cl}]$ 0.839-0.925) compared to that using physiological variables only (AUC $0.837,95 \% \mathrm{Cl} 0.787-0.887, P=0.0073$ ), replicated in the validation cohort $(n=85)$. There was no significant improvement in predicting value of physiological variables plus Lac scene for massive transfusion compared to physiological variables (AUC 0.903 vs 0.895 , $P=0.32$ ). The increased blood lactate level per minute from scene to $E R$ was associated with increased probability for immediate intervention $(P<0.0001)$. Both adding Lac scene to physiological variables and the temporal elevation of blood lactate levels from scene to ER could improve the prediction of the immediate intervention.

Immediate surgical/radiological intervention for hemostasis is a key component of management in hemorrhagic trauma $^{1,2}$. Immediate blood transfusion to maintain hemodynamics and adequate tissue oxygenation is often needed $^{3}$. If the need for hemostatic intervention and transfusion is accurately predictable at the scene from which emergency medical services (EMS) transports a patient to a trauma center, more preparation time to ensure earlier initiation of interventions would be available. Thus, development of a precise predictive algorithm for the need for immediate intervention for hemostasis may contribute to improvement of trauma care and better outcomes.

Predictive algorithms for blood transfusion have been studied in trauma ${ }^{4-6}$. In particular, prediction of the need for massive transfusion, i.e., $\geq 10$ units of packed red blood cells (RBCs) within $24 \mathrm{~h}^{7}$, has been extensively studied $^{4,5}$. Precise predictive algorithms for the need for massive transfusion using physiological variables without blood tests have been well established ${ }^{4,5,8}$. In contrast, less attention has been devoted to development of precise predictive algorithms for the need for immediate hemostatic interventions and transfusion.

The blood lactate level reflects poor tissue perfusion and is used to guide the management of with patients with circulatory disturbance in trauma ${ }^{9}$. Portable devices enable point-of-care lactate testing even in prehospital settings ${ }^{10,11}$. The potential utility of measuring blood lactate levels in prehospital settings was reported in patients with circulatory, respiratory, or neurological disorders who required urgent ambulance dispatch ${ }^{12}$.

\footnotetext{
${ }^{1}$ Senshu Trauma and Critical Care Center, $2-23$ Rinku Orai Kita, Osaka, 598-8577, Japan. ${ }^{2}$ Chiba University Graduate School of Medicine, Department of Emergency and Critical Care Medicine, 1-8-1 Inohana, Chuo, Chiba, 260-8677, Japan. ${ }^{3}$ Center for Frontier Medical Engineering, Chiba University, 1-8-1 Inohana, Chuo, Chiba, 260-8677, Japan. ${ }^{4}$ Shimane University Faculty of Medicine, Department of Acute Care Surgery, 89-1 Enya-cho, Izumo, Shimane, 693-8501, Japan. Correspondence and requests for materials should be addressed toT.-a.N. (email: taka.nakada@nifty.com)
} 
Thus, we hypothesized that blood lactate levels at the scene could improve the prediction of need for immediate interventions for hemorrhage after hospital arrival. We prospectively measured blood lactate levels in trauma patients both at the scene and on arrival at a level I regional trauma center. Primary outcome was immediate intervention for hemorrhage defined as immediate surgical/radiological intervention for hemostasis and/or blood transfusion within $24 \mathrm{~h}$ after emergency room (ER) arrival. The amount of change per minute in blood lactate levels taken at the scene and on ER arrival was further evaluated.

\section{Results}

Baseline patient characteristics and outcomes. In derivation cohort 1, patients in the case group were significantly older, with higher probability of penetrating injury, higher injury severity score, higher blood lactate levels on scene (Lac scene) and in the ER (Lac emergency room), and worse physiological data compared to the control group (Table 1). Patients in the case group had increased 28-day mortality and longer intensive care unit stay compared to the control group $(P<0.0001)$.

Prediction of need for immediate intervention for hemorrhage. In the univariate analysis of immediate need for intervention for hemorrhage, Lac scene had the highest area under the curve (AUC) value (0.764 [95\% confidence interval (CI) 0.698-0.829]) (Table 2A) (Fig. 1A). In the primary analysis, physiological variables plus Lac scene significantly increased the predictive value for the need for immediate intervention for hemorrhage (AUC 0.882 [95\% CI 0.839-0.925]) compared to that using physiological variables only (AUC 0.837 [95\% CI $0.787-0.887]$ ) (physiological variables vs. physiological plus lactate, $P=0.0073$ ) (Table 2B) (Fig. 1B).

In validation analysis for predictive ability in cohort 2 using the cut-off values of the derivation analysis in cohort 1 (Baseline patient characteristics and outcomes, (Table E1, see Additional file 1), the sensitivity and specificity using the combination of physiological variables plus Lac scene were 0.846 and 0.800 , replicating the findings in cohort 1 (sensitivity $=0.833$, specificity $=0.842)$.

Prediction of need for massive transfusion. Univariate analysis in combined cohorts $1+2$ (Baseline patient characteristics and outcomes, (Table E2, see Additional file 1) showed that the GCS score had the highest AUC value for prediction of the need for massive transfusion, followed by the Lac scene value (Table $3 \mathrm{~A}$ ). In multivariate analysis of the need for massive transfusion, the predictive AUC value (0.895 [95\% CI 0.846-0.944]) using physiological variables was already high (Table 3B). There was no significant improvement in predictive value for massive transfusion using physiological variables plus Lac scene compared to that using physiological variables alone (physiological variables vs. physiological variables plus lactate, $P=0.32$ ).

Change in blood lactate levels from scene to ER (Lac delta per min). Patients in the positive Lac delta per min group, with increased blood lactate levels from scene to ER, had significantly increased probability of need for immediate intervention for hemorrhage compared to the negative Lac delta per min group $(P=0.019)$ (Fig. 2A). In the positive Lac delta per min group, the case probability was significantly increased in higher quintiles $(P<0.0001)$ (Fig. 2B).

\section{Discussion}

This study investigated the predictive value of blood lactate levels at the scene for the need for immediate intervention for hemorrhage. We found that adding the blood lactate level at the scene to a combination of physiological variables significantly improved the predictive value. In contrast, the combination of physiological variables without blood lactate levels showed high predictive value for the need for massive transfusion; there was no significant improvement in predictive value using physiological variables plus Lac scene for the need for massive transfusion compared to that using physiological variables alone. However, the increased blood lactate level per minute from scene to ER was associated with increased probability of the need for immediate intervention for hemorrhage.

The potential usefulness of prehospital lactate was suggested as a tool for urgent ambulance dispatch for patients with circulatory, respiratory, or neurological disorders ${ }^{12}$, as well as a predictive tool for resuscitative care $^{13}$ and designation of trauma activation level ${ }^{14}$. Since the prehospital lactate cost per measurement is not large (\$2.00 per measurement) in the study, the study results have high translatability into clinical practices. In addition, lactate data on ER arrival may be useful for initial in-hospital triage to determine which patients need early trauma team activation and surgical intervention ${ }^{15}$. Massive transfusion only occurs in $1 \%$ to $5 \%$ of civilian trauma patients ${ }^{6}$; the rate of immediate intervention for hemorrhage is higher than that for massive transfusion. Furthermore, more deaths occur in patients who receive $<10$ units of packed $\mathrm{RBCs}^{7}$. Thus, we focused on immediate intervention for hemorrhage in the current study. In addition, since predicting which patients will have less hemorrhage may be difficult, developing predictive algorithms with high precision based on data at the earliest phase may improve the quality of subsequent trauma care.

In a study of blood transfusion with $\geq 6$ units of packed RBCs within $24 \mathrm{~h}$, prehospital blood lactate level had a better predictive value than sBP for transfusion requirement (AUC, lactate vs. sBP, 0.72 vs 0.61$)^{16}$. We also found that prehospital blood lactate level had better predictive value for the need for immediate intervention for hemorrhage compared to that using sBP (AUC, lactate vs. sBP, 0.764 vs. 0.568 ). In normotensive trauma patients, there was also a significant association between prehospital lactate and transfusion requirements; however, the predictive value of a single prehospital lactate level was inadequate $(A U C=0.68)$. Thus, a single lactate level may be insufficient to predict the need for transfusion or immediate intervention for hemorrhage. In the primary analysis of the study, we found that the blood lactate level at the scene in combination with physiological variables significantly improved the predictive value (Table $2 \mathrm{~B}$ ). The predictive ability was replicated in validation analysis. The predictive value of physiological variables plus lactate level at the scene was very high (AUC $0.882,95 \%$ CI 0.839-0.925), and highlights the importance of lactate measurement combined with physiological variables. It 


\begin{tabular}{|c|c|c|c|}
\hline Characteristics or outcomes & Case $^{\alpha}(n=78)$ & Control $(n=272)$ & $P$-values \\
\hline Age, years & $62(41-75)$ & $39(21-55)$ & $<0.0001$ \\
\hline Male, n (\%) & $51(65.4)$ & $189(69.5)$ & 0.58 \\
\hline \multicolumn{4}{|l|}{ Mechanism of injury } \\
\hline Penetrating, n (\%) & $5(6.4)$ & $5(1.8)$ & 0.048 \\
\hline Blunt, n (\%) & $73(93.6)$ & $267(98.2)$ & 0.30 \\
\hline Road injury, n (\%) & $57(73.1)$ & $224(82.4)$ & \\
\hline Fall, n (\%) & $10(12.8)$ & $26(9.6)$ & \\
\hline Compression machinery, n (\%) & $4(5.1)$ & $6(2.2)$ & \\
\hline Other, n (\%) & $2(2.6)$ & $11(4.0)$ & \\
\hline \multicolumn{4}{|l|}{ Physiological data $^{\beta}$} \\
\hline Systolic blood pressure, $\mathrm{mm} \mathrm{Hg}$ & $126(101-145)$ & $131(118-146)$ & 0.069 \\
\hline Heart rate, beats/min & $92(80-114)$ & $85(74-97)$ & 0.0014 \\
\hline Respiratory rate, breaths/min & $25(20-30)$ & $21(16-25)$ & 0.0010 \\
\hline Glasgow Coma Scale & $13(8-14)$ & $14(13-15)$ & $<0.0001$ \\
\hline Shock index & $0.75(0.57-1.01)$ & $0.64(0.54-0.77)$ & 0.0003 \\
\hline \multicolumn{4}{|l|}{ Lactate } \\
\hline Scene, mg/dL & $3.1(2.3-4.7)$ & $2.0(1.6-2.6)$ & $<0.0001$ \\
\hline Emergency room, mg/dL & $3.0(2.3-5.0)$ & $1.8(1.3-2.5)$ & $<0.0001$ \\
\hline Delta $^{\dagger}$, delta/min & $0.010(-0.027-0.059)$ & $\begin{array}{l}-0.0071 \\
(-0.028-0.022)\end{array}$ & 0.030 \\
\hline Scene to hospital, min & $22(14-29)$ & $18(13-25)$ & 0.069 \\
\hline Injury severity score & $27(19-40)$ & $9(1-17)$ & $<0.0001$ \\
\hline Positive FAST exam ${ }^{*}, \mathrm{n}(\%)$ & $10(12.8)$ & $5(1.8)$ & 0.0002 \\
\hline Blood transfusion, n (\%) & $65(83.3)$ & - & - \\
\hline Red blood cells ${ }^{\S}, \mathrm{mL}$ & $560(0-1120)$ & - & - \\
\hline Fresh frozen plasma, $\mathrm{mL}$ & $1040(480-2060)$ & - & - \\
\hline Massive transfusion, n (\%) & $28(35.9)$ & - & \\
\hline \multicolumn{4}{|l|}{ Hemostatic intervention } \\
\hline Surgery, n (\%) & $12(15.4)$ & - & - \\
\hline IVR, n (\%) & $28(35.9)$ & - & - \\
\hline Both, n (\%) & $6(7.7)$ & - & - \\
\hline ICU admission, n (\%) & $74(94.9)$ & $250(91.9)$ & 0.53 \\
\hline Length of ICU stay (days) & $12(6-18)$ & $2(2-3)$ & $<0.0001$ \\
\hline 28-day mortality, n (\%) & $10(12.8)$ & $0(0.0)$ & $<0.0001$ \\
\hline
\end{tabular}

Table 1. Baseline patient characteristics and clinical outcomes in cohort 1. IVR, interventional radiology; ICU, intensive care unit; FAST, focused assessment with sonography in trauma. ${ }^{\alpha}$ Cases were defined as patients who required immediate intervention for hemorrhage. Immediate intervention was defined as immediate surgical/ radiological intervention for hemostasis and/or blood transfusion for traumatic hemorrhage within $24 \mathrm{~h}$ after emergency room arrival. ${ }^{\beta}$ Data for lactate were obtained at the scene. ${ }^{\dagger}$ Delta value for lactate was calculated with the following formula: (lactate in the emergency room - lactate at the scene)/time from scene to hospital. ${ }^{*}$ Examination was performed at the scene. ${ }^{\S}$ Total volume within $24 \mathrm{~h}$ of emergency room arrival. 'Transfusion with $\geq 10$ units of packed red blood cells. Data are presented as median and interquartile range for continuous variables. $P$ values were calculated using Pearson's chi-square test, Fisher's exact test, or the Mann-Whitney $U$ test.

was reported that the assessment of blood consumption (ABC) score, which only includes sBP, HR, a positive focused assessment with sonography in trauma (FAST) and a penetrating mechanism of injury without blood test, have high predictive value for the need for massive transfusion (AUC, 0.83-90) ${ }^{4,5,9}$. This study similarly found that use of physiological variables alone had a very high predictive value (AUC 0.895) (Table 3B). Thus, physiologic parameters may have sufficiently high predictive value for the need for massive transfusion. In addition, we found no significant improvement in predictive value with use of physiological variables plus Lac scene for the need for massive transfusion compared to that using physiological variables alone. Another study similarly showed that either continuous pulse oximetry or prothrombin time could be more useful in prediction of the need for massive transfusion than lactate levels ${ }^{17}$. Furthermore, a predictive model using 15-min continuous vital sign data had high predictive value for the need for massive transfusion (AUC 0.91$)^{18}$. Thus, continuous monitoring of physiologic parameters may have better predictive value for the need for massive transfusion.

The utility of blood lactate measurement in the ER for patients with trauma and sepsis has been widely recognized $^{19,20}$. Delta lactate, also called lactate clearance, is useful in evaluating the trend in circulatory failure in trauma ${ }^{21}$ and sepsis ${ }^{22}$. In this study, the positive Lac delta per min group had a significantly increased case probability compared to that in the negative Lac delta per min group. In addition, in the positive Lac delta per min group, the case probability was significantly increased in higher quintiles. Thus, in addition to the use of 


\begin{tabular}{|l|l|l|l|l|l|}
\hline A. Univariate & AUC $(\mathbf{9 5} \% \mathbf{C I})$ & $P$ value & Cut-off value & Sensitivity, specificity & OR (95\% CI) \\
\hline Variable & $0.764(0.698-0.829)$ & $<0.0001$ & 2.8 & $0.628,0.801$ & $6.82(6.54-7.10)$ \\
\hline Lactate level at the scene & $0.568(0.486-0.649)$ & 0.22 & 101 & $0.269,0.926$ & $4.64(4.30-4.99)$ \\
\hline Systolic blood pressure & $0.619(0.544-0.694)$ & 0.019 & 112 & $0.282,0.919$ & $4.46(4.13-4.80)$ \\
\hline Heart rate & $0.621(0.544-0.699)$ & 0.020 & 24 & $0.667,0.632$ & $3.44(3.17-3.71)$ \\
\hline Respiratory rate & $0.744(0.682-0.806)$ & $<0.0001$ & 13 & $0.628,0.717$ & $4.28(4.01-4.55)$ \\
\hline Glasgow Coma Scale & $0.634(0.556-0.712)$ & 0.010 & 0.90 & $0.359,0.893$ & $4.69(4.39-4.80)$ \\
\hline Shock index & AUC (95\% CI) & P value & & Sensitivity, specificity & OR (95\% CI) \\
\hline B. Multivariate & $0.837(0.787-0.887)$ & $<0.0001$ & & $0.795,0.776$ & $13.03(12.71-13.34)$ \\
\hline Variable & $0.882(0.839-0.925)$ & $<0.0001$ & & $0.833,0.842$ & $25.60(25.26-25.94)$ \\
\hline Physiological variables ${ }^{\alpha}$ & & &
\end{tabular}

Table 2. Receiver operating characteristic curve analysis for prediction of need for blood transfusion or hemostatic intervention using each factor at the scene in cohort 1. AUC, area under the curve; CI, confidence interval; OR, odds ratio. ${ }^{\alpha}$ Physiological variables include systolic blood pressure, heart rate, respiratory rate, Glasgow Coma Scale score, shock index score at the scene, and mechanism of penetrating injury.
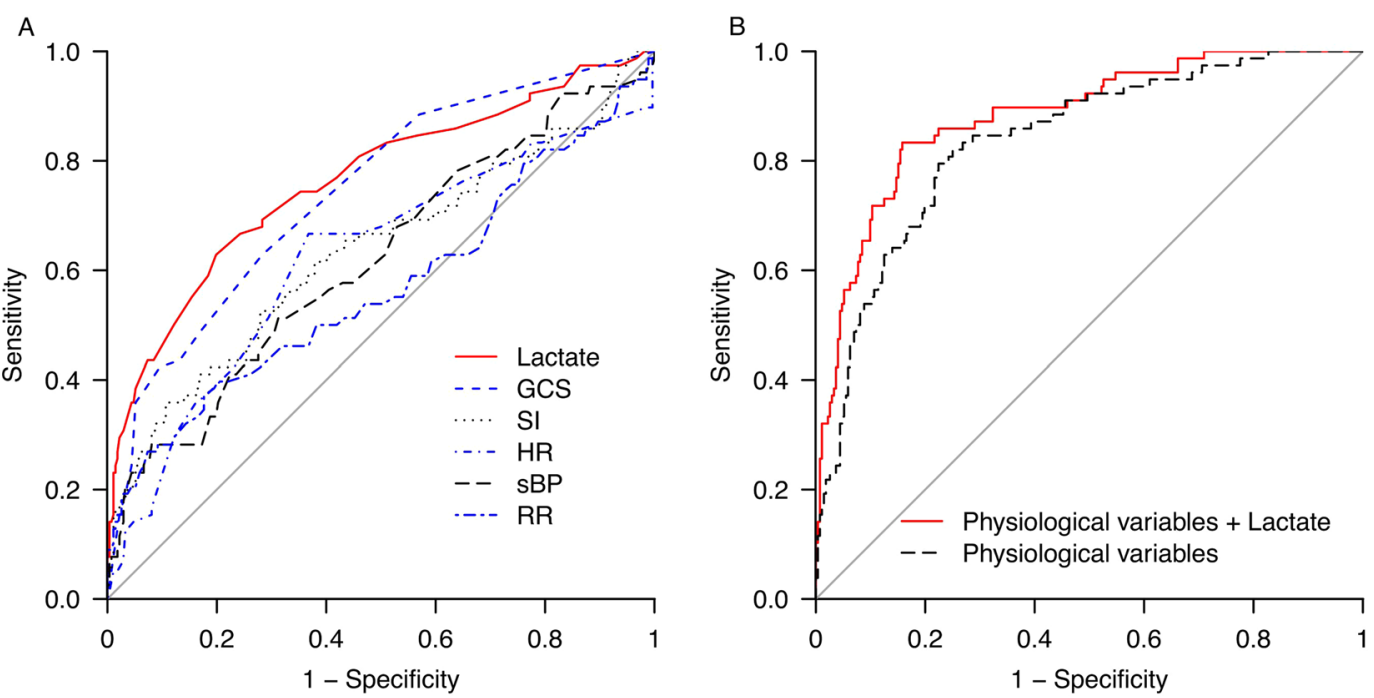

Figure 1. Receiver operating characteristic curve for prediction of immediate need for intervention for hemorrhage. The value of a model using potential predictors of the need for immediate intervention for hemorrhage was determined. Each physiologic parameter (sBP, HR, RR, GCS, and SI) and Lac scene was used for prediction in univariate analysis $(\mathbf{A})$. Lac scene had the greatest predictive value $(\mathrm{AUC}=0.764)$, followed by GCS (AUC $=0.744)$. The predictive ability of a model using a combination of physiological parameters and a penetrating mechanism of injury, with or without Lac scene, was estimated using multivariate analysis (B). Compared with use of parameters without Lac scene $(\mathrm{AUC}=0.837)$, a combination of physiological parameters, a penetrating mechanism of injury, and Lac scene enabled more accurate prediction (AUC $=0.882$ ).

prehospital lactate at the scene for prediction of the need for immediate intervention, assessing the amount of change on ER arrival may detect patients who had bleeding during transfer and need immediate intervention for hemorrhage.

This study had several limitations. While demonstrating the significant value of blood lactate levels at the scene, this observational study was retrospectively conducted at a single level I trauma center. Although there was a significant association between Lac delta per min and altered case probability, the transfer time from scene to hospital was approximately $20 \mathrm{~min}$, due to the urban location. Further studies in different settings may strengthen the findings for the value of Lac delta per min.

\section{Conclusions}

Adding blood lactate levels at the scene to a predictive algorithm using physiological variables significantly improves the predictive value of the need for immediate intervention for hemorrhage. The increase in blood lactate levels from scene to ER was associated with a high probability of the need for immediate intervention. Measuring blood lactate level at the scene appears to be a useful option in trauma care. 


\begin{tabular}{|l|l|l|l|l|l|}
\hline A. Univariate & AUC $(\mathbf{9 5} \% \mathbf{C I})$ & P value & Cut-off value & Sensitivity, specificity & OR (95\% CI) \\
\hline Variable & $0.764(0.661-0.867)$ & $<0.0001$ & 3.1 & $0.647,0.835$ & $9.08(8.70-9.46)$ \\
\hline Lactate & $0.713(0.607-0.819)$ & 0.00073 & 93 & $0.441,0.955$ & $16.48(16.06-4.01)$ \\
\hline Systolic blood pressure & $0.651(0.539-0.764)$ & 0.023 & 100 & $0.529,0.766$ & $3.65(3.29-4.01)$ \\
\hline Heart rate & $0.530(0.406-0.654)$ & 0.73 & 24 & $0.618,0.576$ & $2.16(1.80-2.52)$ \\
\hline Respiratory rate & $0.796(0.718-0.874)$ & $<0.0001$ & 8 & $0.559,0.910$ & $12.60(12.22-12.98)$ \\
\hline Glasgow Coma Scale & $0.763(0.663-0.862)$ & $<0.0001$ & 0.95 & $0.529,0.910$ & $11.23(10.85-11.61)$ \\
\hline Shock index & AUC (95\% CI) & P value & & Sensitivity, specificity & OR (95\% CI) \\
\hline B. Multivariate & $0.895(0.846-0.944)$ & $<0.0001$ & & $0.853,0.820$ & $24.38(23.89-24.86)$ \\
\hline Variable & $0.903(0.851-0.956)$ & $<0.0001$ & & $0.941,0.748$ & $38.48(37.82-39.15)$ \\
\hline Physiological variables ${ }^{\alpha}$ & & &
\end{tabular}

Table 3. Receiver operating characteristic curve analysis for prediction of need for massive transfusion using each factor at the scene in cohort $1+2$. AUC, area under the curve; CI, confidence interval; OR, odds ratio. ${ }^{\alpha}$ Physiological variables include systolic blood pressure, heart rate, respiratory rate, Glasgow Coma Scale score, shock index score at the scene, and positive mechanism of penetrating injury.

A

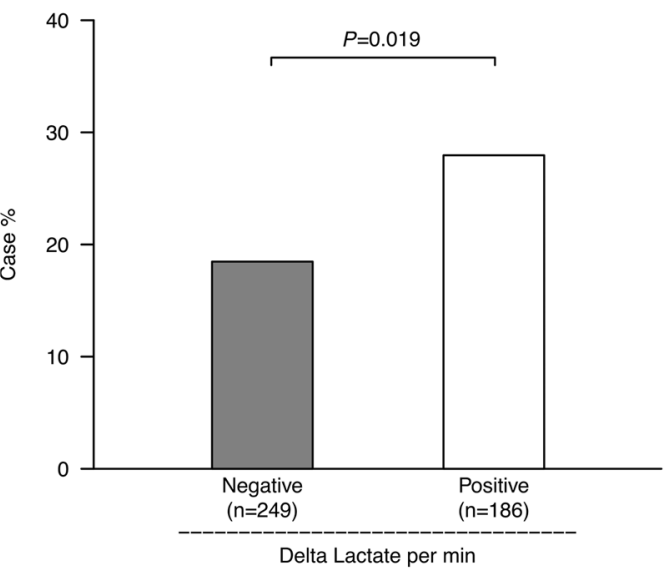

B

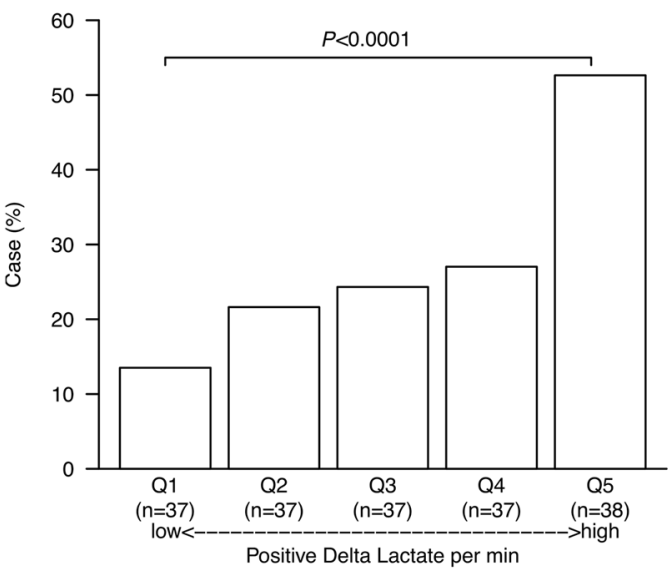

Figure 2. Case probability of patients with early therapeutic intervention in Lac delta per min groups. There was a significant difference in case probability between the negative Lac delta per min group $(\mathrm{n}=249)$ and the positive Lac delta per min group $(\mathrm{n}=186)(\mathrm{A} * P=0.019$ with the chi-square test). In quintile subgroups of the positive Lac delta per min group, the case probability was significantly increased with increasing Lac delta per $\min (\mathbf{B} * * P<0.0001$ with the Cochran-Armitage test).

\section{Methods}

The institutional review board at Senshu Trauma and Critical Care Center approved this study. The methods were carried out in accordance with the Declaration of Helsinki and the relevant guidelines. The review board waived the need for written informed consent from subjects or their legal surrogates.

Study setting and patients. We prospectively measured blood lactate levels and assessed retrospectively. However, the current observational study was retrospectively conducted and may have potential biases.

The Senshu Trauma and Critical Care Center is a level I regional trauma center located in an urban area, Osaka, Japan, and covers about 1 million residents within a 40-km radius. The trauma center has a fully-equipped ambulance to provide advanced prehospital care by trained trauma physicians $24 / 7$. When regional 911 centers receive trauma calls that meet predetermined criteria for severe injury, the emergency ambulance is dispatched, staffed with 2 trained trauma physicians, 1 nurse, 1 EMS technician, and a driver ${ }^{23}$.

Consecutive trauma patients $(n=1,695)$ who were transferred to the center between April 2014 and September 2017 were screened. Of these, 616 patients who were transferred via the trauma physician-staffed ambulance were included. Of these, 94 patients with seizures, hypothermia (below $35.0^{\circ} \mathrm{C}$ ), acute alcohol/drug intoxication, or cardiac arrest were excluded, as these conditions potentially increase blood levels of lactate. Of the remaining 522 patients, 72 without data for blood lactate levels were excluded. Thus, 435 patients were eligible for the analysis (Fig. E1, see Additional file 1).

To demonstrate robustness of the present study findings, we categorized subjects into a derivation cohort 1 or validation cohort 2 . According to a sample size calculation based on area under the curve (AUC) values in previous trauma studies on transfusion $(\mathrm{AUC}=0.80 \text {, power }=0.95 \text {, significance }=0.05 \text {, pROC package for } \mathrm{R})^{4,17,24}$, 
the required sample size in a validation cohort was $n=20$ per group. To obtain the required sample size in a validation cohort, study subjects were randomly divided into a derivation cohort 1 (total $n=350$; case $=78$, control $=272$ ) and a validation cohort 2 (total $n=85$; case $=20$, control $=65$ ) using the rand function of MATLAB software package (version 8.3.0.532 (R2014a), MathWorks, Inc., Natick, MA, USA).

Data collection and definition. Physiologic data collected by a trained trauma team (nurse and physician) on scene included: systolic blood pressure (sBP), heart rate (HR), respiratory rate (RR), Glasgow Coma Scale (GCS) score, and shock index (SI) calculated with sBP divided by HR.

Blood levels of lactate at the scene (Lac scene) were immediately measured after arrival using a portable device (Lactate Pro, Arkray, Kyoto, Japan). The test strip fills with $5 \mu \mathrm{L}$ of whole blood through capillary action and takes $60 \mathrm{~s}$ to provide the lactate level. The cost of test strip per measurement is approximately $\$ 2.00$. A drop of venous blood from a peripheral catheter immediately after insertion and before connection of infusion fluid was used for lactate measurement. Blood levels of lactate in the ER (Lac emergency room) were measured immediately after arrival at the trauma center using a blood gas analyzer (ABL800, Radiometer, Copenhagen, Denmark). A strong correlation between blood levels of lactate using the two devices was previously reported ${ }^{25}$.

The change in amount of blood lactate per minute between Lac emergency room and Lac scene was defined as Lac delta per min: (Lac emergency room - Lac scene) / time in minutes from medical team arrival at the scene to emergency room arrival. The "negative Lac delta per min group" was defined by Lac delta per min $<0$ and the "positive Lac delta per min group" was defined by Lac delta per min $\geq 0$.

The primary outcome variable was immediate intervention for hemorrhage, defined as immediate surgi$\mathrm{cal} /$ radiological intervention for hemostasis and/or blood transfusion. Immediate hemostatic intervention was defined as intervention immediately after assessment in the ER and included either surgical or radiological intervention for hemostasis. Immediate blood transfusion was defined as blood transfusion for traumatic hemorrhage within $24 \mathrm{~h}$ after ER arrival. We categorized patients with immediate interventions for hemorrhage into the case group and those without interventions into the control group.

The secondary outcome variable was massive transfusion. Massive transfusion was defined as $\geq 10$ units of packed RBCs in the first $24 \mathrm{~h}^{8}$.

Statistical analysis. Each of the physiological variables (sBP, HR, RR, GCS, and SI) and Lac scene was analyzed as a determinant with univariate logistic regression in cohort 1 . Multivariate logistic regression analysis was simultaneously performed. A penetrating mechanism of injury was reported in predictive algorithms for transfusion $^{4}$, and was added in the multivariate analysis as a covariate. The predictive ability of each model was evaluated with a receiver operating characteristic (ROC) curve and corresponding AUC, which were derived through leave-one-out cross-validation. We determined the optimal cut-off points with the Youden index to estimate the sensitivity, specificity, and odds ratio for the factors in each regression model.

For primary analysis of the study, we compared the ROC-AUC results for physiological variables only with physiological variables plus Lac scene using the DeLong test. To validate the results in cohort 1 , we analyzed the sensitivity, specificity, and odds ratio (OR) in combination with the physiological variables and lactate in cohort 2 using the cut-off points of cohort 1.

A secondary analysis in the study assessed need for massive transfusion. The predictive ability for massive transfusion was analyzed using the same methods for immediate intervention for hemorrhage. Due to a small sample size, we combined cohorts 1 and 2 to analyze use of massive transfusion.

Another secondary analysis examined Lac delta per min. We first compared the case probability between negative and positive Lac delta per min groups using the chi-square test. We further compared the case probability among quintile subgroups in the positive Lac delta per min group using the Cochran-Armitage test.

We tested for differences in baseline characteristics using the chi-square test or Fisher's exact test for categorical data and the Mann-Whitney $U$ test for continuous data. Data were expressed as median (interquartile range $[\mathrm{IQR}]$ ) for continuous values and absolute number and percentage for categorical values. A two-tailed $P$ value $<0.05$ was considered significant. Analyses were performed using R (version 3.3.2, www.R-project.org) and pROC package for $\mathrm{R}$ was used for sample size calculation.

Ethics approval and consent to participate. The institutional review board at Senshu Trauma and Critical Care Center approved this study. The methods were carried out in accordance with the Declaration of Helsinki and the relevant guidelines. The review board waived the need for written informed consent from subjects or their legal surrogates.

\section{Data Availability}

The datasets used and analyzed during our study are available from the corresponding author upon reasonable request.

\section{References}

1. Remick, K. N. et al. Defining the optimal time to the operating room may salvage early trauma deaths. J Trauma Acute Care Surg. 76, 1251-1258 (2014).

2. Matsushima, K. et al. Effect of door-to-angioembolization time on mortality in pelvic fracture: Every hour of delay counts. J Trauma Acute Care Surg. 84, 685-692 (2018).

3. Powell, E. K. et al. Shorter times to packed red blood cell transfusion are associated with decreased risk of death in traumatically injured patients. J Trauma Acute Care Surg. 81, 458-462 (2016).

4. Nunez, T. C. et al. Early prediction of massive transfusion in trauma: simple as ABC (assessment of blood consumption)? J Trauma. 66, 346-352 (2009). 
5. Rau, C. S. et al. Prediction of Massive Transfusion in Trauma Patients with Shock Index, Modified Shock Index, and Age Shock Index. Int J Environ Res Public Health. 13, E683 (2016).

6. Holcomb, J. B. et al. Damage control resuscitation: directly addressing the early coagulopathy of trauma. J Trauma. 62, 307-310 (2007).

7. Como, J. J., Dutton, R. P., Scalea, T. M., Edelman, B. B. \& Hess, J. R. Blood transfusion rates in the care of acute trauma. Transfusion. 44, 809-813 (2004).

8. Estebaranz-Santamaría, C., Palmar-Santos, A. M. \& Pedraz-Marcos, A. Massive transfusion triggers in severe trauma: Scoping review. Rev Lat Am Enfermagem. 26, e3102 (2018).

9. Chaochankit, W., Akaraborworn, O., Sangthong, B. \& Thongkhao, K. Combination of blood lactate level with assessment of blood consumption (ABC) scoring system: A more accurate predictor of massive transfusion requirement. Chin J Traumatol. 21, 96-99 (2018).

10. Regnier, M. A. et al. Prognostic significance of blood lactate and lactate clearance in trauma patients. Anesthesiology. 117, 1276-1288 (2012).

11. Shapiro, N. I. et al. Serum lactate as a predictor of mortality in emergency department patients with infection. Ann Emerg Med. 45, 524-528 (2005).

12. Jansen, T. C. et al. The prognostic value of blood lactate levels relative to that of vital signs in the pre-hospital setting: a pilot study. Crit Care. 12, R160 (2008).

13. Guyette, F. X. et al. A comparison of prehospital lactate and systolic blood pressure for predicting the need for resuscitative care in trauma transported by ground. J Trauma Acute Care Surg. 78, 600-606 (2015).

14. Brown, J. B. et al. Prehospital lactate improves accuracy of prehospital criteria for designating trauma activation level. J Trauma Acute Care Surg. 81, 445-452 (2016).

15. Caputo, N. et al. Triage vital signs do not correlate with serum lactate or base deficit, and are less predictive of operative intervention in penetrating trauma patients: a prospective cohort study. Emerg Med J. 30, 546-550 (2013).

16. Vandromme, M. J., Griffin, R. L., Weinberg, J. A., Rue, L. W. 3rd \& Kerby, J. D. Lactate is a better predictor than systolic blood pressure for determining blood requirement and mortality: could prehospital measures improve trauma triage? J Am Coll Surg. 210, 861-867 (2010).

17. Shackelford, S. et al. Predicting blood transfusion using automated analysis of pulse oximetry signals and laboratory values. $J$ Trauma Acute Care Surg. 2015; 79, S175-180 (2015).

18. Parimi, N. et al. Automated continuous vital signs predict use of uncrossed matched blood and massive transfusion following trauma. J Trauma Acute Care Surg. 80, 897-906 (2016).

19. Parsikia, A. et al. The predictive value of initial serum lactate in trauma patients. Shock. 42, 199-204 (2014).

20. Karon, B. S. et al. Evaluation of lactate, white blood cell count, neutrophil count, procalcitonin and immature granulocyte count as biomarkers for sepsis in emergency department patients. Clin Biochem. 50, 956-958 (2017).

21. Dezman, Z. D. et al. Failure to clear elevated lactate predicts 24-hour mortality in trauma patients. J Trauma Acute Care Surg. 79, 580-585 (2015).

22. Ryoo, S. M. et al. Lactate Level Versus Lactate Clearance for Predicting Mortality in Patients With Septic Shock Defined by Sepsis-3. Crit Care Med. 46, e489-e495 (2018).

23. Nakada, T. A. et al. Development of a prehospital vital signs chart sharing system. Am J Emerg mMed. 34, 88-92 (2016).

24. Mackenzie, C. F. et al. Automated prediction of early blood transfusion and mortality in trauma patients. J Trauma Acute Care Surg. 76, 1379-1385 (2014)

25. Sloet van Oldruitenborgh-Oosterbaan, M. M., van den Broek, E. T. \& Spierenburg, A. J. Evaluation of the usefulness of the portable device Lactate Pro for measurement of lactate concentrations in equine whole blood. J Vet Diagn Invest. 20, 83-85 (2008).

\section{Author Contributions}

Study concept and design: Nakada. Acquisition of data: Fukuma, Nakada, Nakao, Watanabe, Mizushima, Matsuoka ${ }^{1}$. Drafting of the manuscript: Nakada, Shimada, Shimazui, Aizimu. Critical revision of the manuscript for important intellectual content: Fukuma, Nakada, Shimada, Shimazui, Aizimu, Nakao, Watanabe, Mizushima, Matsuoka. Statistical analysis: Nakada, Aizimu, Supervision: Nakada. All authors read and approved the final manuscript.

\section{Additional Information}

Supplementary information accompanies this paper at https://doi.org/10.1038/s41598-019-50253-6.

Competing Interests: The authors declare no competing interests.

Publisher's note Springer Nature remains neutral with regard to jurisdictional claims in published maps and institutional affiliations.

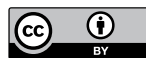

Open Access This article is licensed under a Creative Commons Attribution 4.0 International

License, which permits use, sharing, adaptation, distribution and reproduction in any medium or format, as long as you give appropriate credit to the original author(s) and the source, provide a link to the Creative Commons license, and indicate if changes were made. The images or other third party material in this article are included in the article's Creative Commons license, unless indicated otherwise in a credit line to the material. If material is not included in the article's Creative Commons license and your intended use is not permitted by statutory regulation or exceeds the permitted use, you will need to obtain permission directly from the copyright holder. To view a copy of this license, visit http://creativecommons.org/licenses/by/4.0/.

(C) The Author(s) 2019 